\title{
Ameliorating the antitumor activity of lenalidomide using PLGA nanoparticles for the treatment of multiple myeloma
}

\author{
Veera Venkata Satyanarayana Reddy Karri', Nagasamy Venkatesh Dhandapani ${ }^{1}$, Sai Sandeep \\ Mannemala $^{2,3}$, Kollipara Radhakrishna ${ }^{1}$, Shashank Mulukutla ${ }^{4}$, Dedeepya Sudunagunta ${ }^{2}$
}

\begin{abstract}
${ }^{1}$ Department of Pharmaceutics, JSS College of Pharmacy, Ootacamund, JSS University, Mysore, India, ${ }^{2}$ Department of Pharmaceutical Analysis, JSS College of Pharmacy, Ootacamund, JSS University, Mysore, India, ${ }^{3}$ Department of Pharmacy, Annamalai University, Annamalai Nagar, Tamil Nadu, India, ${ }^{4}$ Department of Pharmacology, JSS College of Pharmacy,
\end{abstract}

Ootacamund, JSS University, Mysore, India

\begin{abstract}
Lenalidomide (LND) is an anti-cancer drug and an effective derivative of thalidomide used for multiple myeloma therapy. Because of its poor solubility in water, LND is known to cause low oral bioavailability (below 33\%), and as a direct consequence of this, the dosing frequency is extended thus increasing risk of toxicity. To improve its bioavailability and sustained release, the present study aims to formulate polymeric nanoparticles (NPs) for LND using [Poly (lactic-co-glycolic acid)] (PLGA) as a polymer. The polymeric NPs were evaluated for particle size, SEM, XRD, drug content, entrapment efficiency (EE), in vitro release studies and in vivo bioavailability studies in rats. The formulated NPs possessed a size of $179 \pm 0.9 \mathrm{~nm}$ and a zeta potential of $-24.4 \pm 0.2 \mathrm{mV}$. The drug loading and EE of the optimized formulation was $32 \pm 0.37 \%$ and $78 \pm 0.92 \%$ respectively. After oral administration of LND PLGA-NPs, the relative bioavailability was enhanced about 3.67-fold compared to LND. This study demonstrates the novel drug delivery for LND with PLGA-NPs as effective drug delivery system for sustained delivery of LND.
\end{abstract}

Uniterms: Multiple myeloma/treatment. Lenalidomide/uses. PLGA/sustained release. Polymeric nanoparticles/development. Solubility.

\section{INTRODUCTION}

Classified as a plasma cell malignancy, multiple myeloma (MM) is identified by the bone marrow accumulation of terminally differentiated B cells. Irrespective of therapeutic progression, MM remains without a cure till date. (Morgenroth et al., 2011). Lenalidomide (LND), a thalidomide analogue is an immunomodulatory agent with antineoplastic and antiangiogenic properties which has been cleared for clinical application for the treatment of MM and transfusion dependent anemia (Kastritis, Dimopoulos, 2007; Richardson et al., 2006). LND is off-white to pale yellow powder marketed with the trade name of Revlimid. Revlimid hemihydrate (commercial form) has poor oral bioavailability $(<33 \%)$ because of its inadequate solubility in water. LND has a shorter half-life of 3 hours

\footnotetext{
*Correspondence: N. V. Dhandapani. JSS College of Pharmacy, Ootacamund. JSS University, Mysore - 643001 - India. E-mail: nagasamyvenkatesh@gmail.com
}

(Song et al., 2014). Novel therapeutic approaches are, therefore, urgently needed. Hence, the main target of this study was to formulate a nanoparticulated drug delivery system for LND which can enhance the solubility of LND, consequently improving its bioavailability.

In the past few decades, nanoparticulated drug delivery systems have remarkably ameliorated the efficacy of conventional therapies, by increasing the solubility and decreasing the drug associated systemic toxicities (Maeda et al., 2000). Selective targeting and preferential accumulation of the drug at the tumor site can be achieved with nanoparticles existing within a diameter range of 20-200 nm, via greater permeability and retention (Egusquiaguirre et al., 2012). PLGA is a biodegradable and biocompatible polymer that is hydrolytically degraded into nontoxic oligomer and monomer, lactic acid, and glycolic acid (Vega et al., 2008). The higher surface area to volume ratio of PLGANPs provides an amended pharmacokinetic and biodistribution profile of the therapeutic agents and thus reduces toxicity due to their specific aggregation at the target site (Noori Koopaei et al., 2014). They also improve 
the solubility of hydrophobic compounds. Therefore, we expect that PLGA-NPs drug delivery system can improve poor oral bioavailability of LND along with sustained release to achieve intended therapeutic effect on level with the marketed products, while keeping its anti-neoplastic activity constant. The ultimate goal of this delivery system is to ensure the applicability to a wider population of cancer patients.

\section{MATERIAL AND METHODS}

\section{Material}

Lenalidomide (98.7\%), and thalidomide (99.1\%) (internal standard, IS) were obtained as a gift sample from NatcoPharmaLtd., (Hyderabad, India). PLGA, tetrahydrofuran, acetonitrile and acetone of HPLC grade was purchased from Sigma Aldrich, (Mumbai, India). The HPLC grade water was obtained by using Milli-Q Academic system, Millipore (Bangalore, India). All other chemicals used in this study were of analytical grade.

\section{Methods}

\section{Preparation of lenalidomide nanoparticles (Venkatesh et al., 2015)}

The nanoprecipation technique was the method of choice for the preparation of the nanoparticles as previously described (Venkatesh et al., 2015). Briefly, LND (20 mg) and PLGA (100 mg) were dissolved in dichlromethane ( $5 \mathrm{~mL}$; Solution A) and were subjected to sonication for $5 \mathrm{~min}$ to dissolve all substances. $125 \mathrm{mg}$ of Poloxamer F-127 was dissolved in $50 \mathrm{~mL}$ of deionized water (Solution B). Solution A was then incorporated into solution $B$ under magnetic stirring at varying rpm using a syringe with a flow rate of $1 \mathrm{~mL} / 10 \mathrm{~min}$. The schematic representation of fabrication of LND-PLGANPs is shown in Figure 1. The obtained nanosuspension was centrifuged, and lyophilized with cryoprotectant ( $2 \%$ sucrose) and tested for various characterization parameters.

\section{Drug loading and entrapment efficiency}

Drug loading and entrapment efficiency are assessed after freeze-drying and adding $10 \mathrm{~mL}$ of acetonitrile (a common solvent for drug and PLGA) to facilitate the coat of the NPs to get dissolved. The obtained suspension was diluted appropriately with mobile phase to determine drug content and entrapment efficiency. Samples were measured at an absorbance of $220 \mathrm{~nm}$ using high pressure liquid chromatography (Figure 2). The following equations

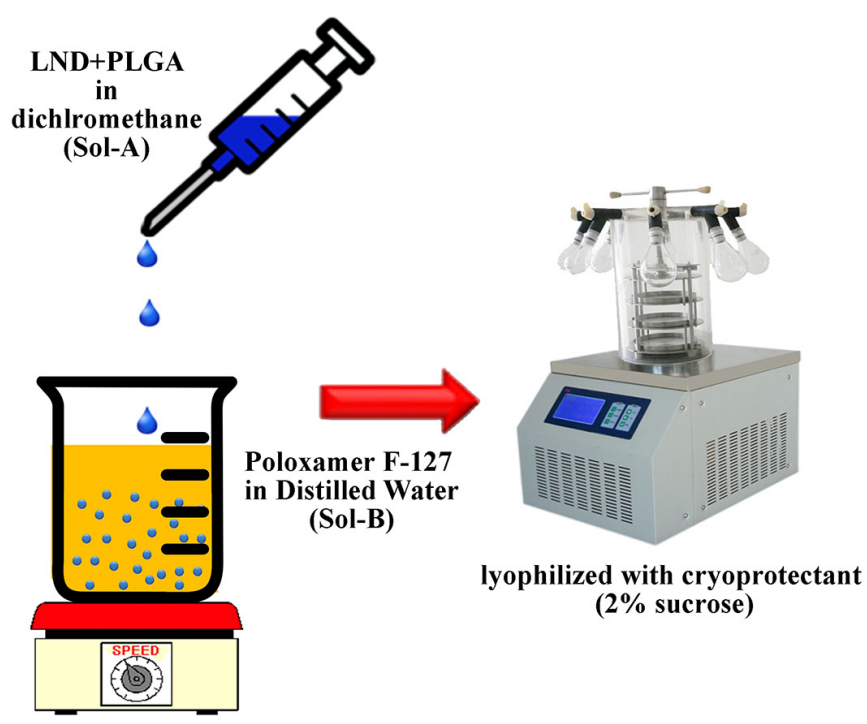

FIGURE 1 - Schematic representation of preparation of lenalidomide-PLGA-NPs.

were utilized for the determination of drug loading and entrapment efficiency of LND in NPs.

Drug loading $(\% w / w)=\frac{\begin{array}{c}\text { Mass of drug in } \\ \text { nanoparticle }\end{array}}{\begin{array}{c}\text { Mass of nanoparticle } \\ \text { recovered }\end{array}} \times 100$

Entrapment efficiency $(\%)=\frac{\begin{array}{c}\text { Mass of the drug in } \\ \text { nanoparticle }\end{array}}{\begin{array}{c}\text { Mass of the drug used } \\ \text { in the formulation }\end{array}} \times 100$

\section{Characterization of nanoparticles}

Particle size and zetapotential (Karri et al., 2015)

The prepared nanoparticles were washed with double distilled water (filtered through $0.22 \mu \mathrm{m}$ ) several times before particle size analysis. The average particle size and zeta potential of the LND-PLGA-NPs were determined by Particle Size Analyzer (Malvern Instruments Ltd, UK) which allows sample measurement in the range of

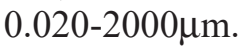

\section{Scanning electron microscopy (SEM)}

The uniformity of the particles (size and shape) were verified using SEM. Lyophilized NPs were re-suspended in distilled water; subsequently they were placed on a silicon grid and left to dry at room temperature. The NPs suspension was then vacuum coated with gold for 3 min before SEM analysis. Surface characteristics of the 

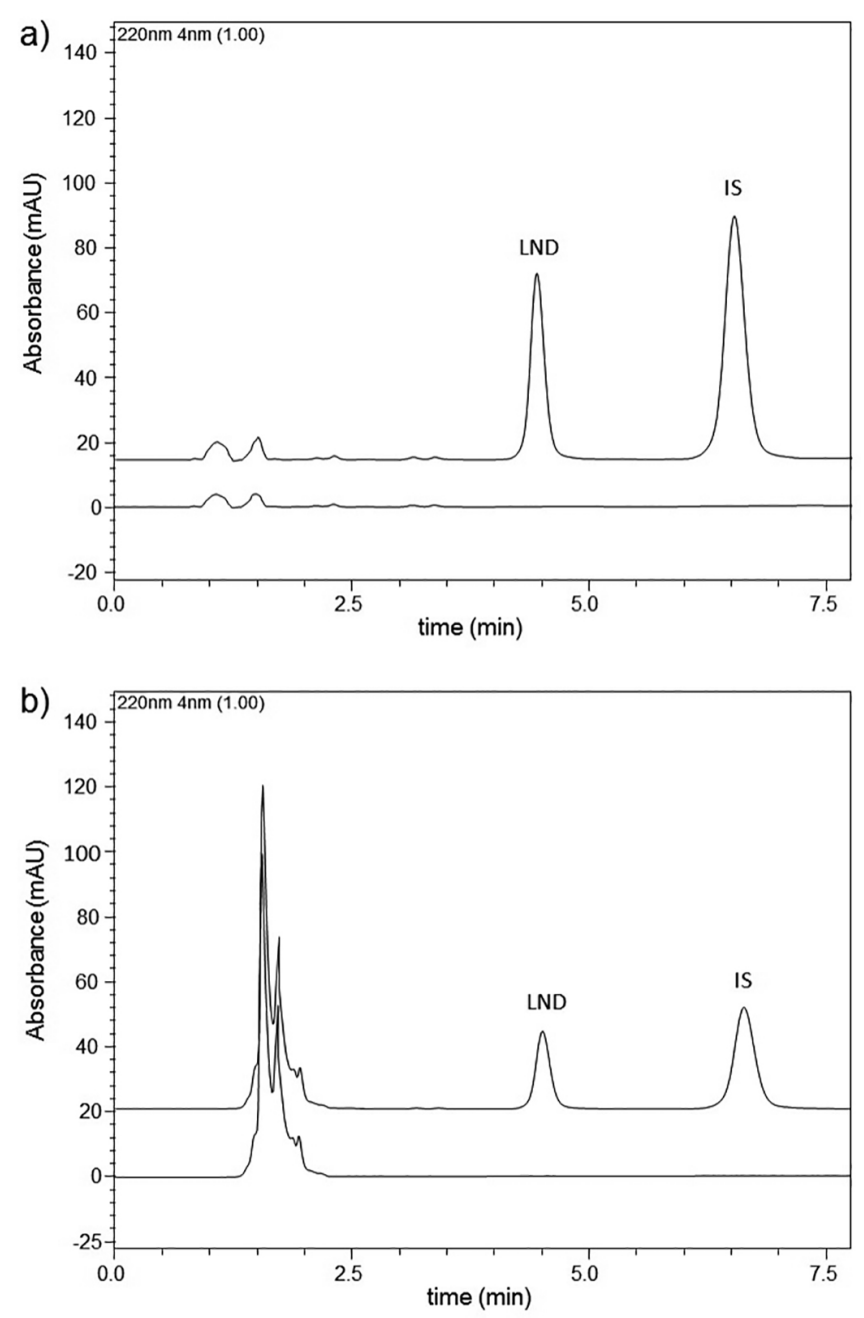

FIGURE 2 -Representative chromatograms corresponding to (a) lenalidomide loaded PLGA-NPs with IS (thalidomide) and blank PLGA-NPs and (b) lenalidomide spiked in rat plasma with IS (thalidomide) and processed blank rat plasma.

samples were observed under a SEM (JEOL Ltd, Japan) operated at $15-\mathrm{keV}$ pulse under different resolutions.

\section{Differential scanning calorimetry}

DSC analysis was performed using DSC Q200. (TA instruments, U.S.A). The samples sealed in aluminum pans and heated at a rate of $10^{\circ} \mathrm{C} \mathrm{per} / \mathrm{min}$ within a temperature range of 30 to $300{ }^{\circ} \mathrm{C}$ with constant nitrogen gas supply at a rate of $40 \mathrm{~mL} / \mathrm{min}$. DSC analysis was performed for PLGA, LND and LND-PLGA nanoparticles.

\section{$X$-Ray diffraction (XRD) studies}

An XRD peak mainly depends on the crystal size as they indicate the crystalline nature at particular value at $2 \theta$ range. Molecular arrangements of LND alone and in nanoparticulate formulations were performed on an X-ray diffractometer (PANalytical X'Pert Pro, The Netherlands) by applying $\mathrm{CuK} \alpha$ radiation. The data was collected with an angular range from $3^{\circ}$ to $50^{\circ} 2 \theta$ in continuous mode using a step size of $0.02^{\circ} 2 \theta$ and step time of $5 \mathrm{sec}$.

\section{ANALYTICAL CONDITIONS}

Quantification of LND in NPs and plasma samples was achieved using Shimadzu HPLC (LC 20 AD) (Kyoto, Japan) connected to a PDA detector (SPD-M20A). The chromatographic separation was performed on a Luna $\mathrm{C}_{18}$ column $(150 \times 4.6 \mathrm{~mm})($ Phenomenex, USA)with a mobile phase containing $20 \mathrm{mM} \mathrm{KH}_{2} \mathrm{PO}_{4}$ : acetonitrile $(86: 14 \mathrm{v} / \mathrm{v})$ at a $\mathrm{pH}$ of 4.2 , supplied at a flow rate of $1 \mathrm{~mL} / \mathrm{min}$. Sample detection was carried out at $220 \mathrm{~nm}$. Data collection and instrumental control was achieved by means of LC Solutions software (SP 1.1). Thalidomide was used as internal standard (IS), as it belongs to same class of LND and offered acceptable resolution with LND peak. Retention times of LND and IS were 4.5and 6.7 min respectively. The calibration curves were linear in the range of $0.5-30 \mu \mathrm{g} / \mathrm{mL}(\mathrm{r}=0.997)$ in NPs, and $20-600 \mathrm{ng} / \mathrm{mL}(\mathrm{r}=0.998)$ in rat plasma, suggesting that the method was linear over the selected range (Mannemala, Nagarajan, 2015b). The intra and inter-day accuracy and precision were within $\mathrm{CV} \% \leq 6 \%$, indicating the method meets the acceptance criteria (FDA, 2001). The extraction efficiency in case of spiked plasma samples was $98.7 \pm 3.1 \%$, suggesting that the procedure was consistent and robust (Mannemala, Nagarajan, 2015a).

In vitro release studies (Gomathi et al., 2014)

The dialysis bag diffusion technique was incorporated to analyze the in vitro drug release of LND from NPs. The drug loaded NPs were placed in the dialysis bag $(3 \mathrm{~mL})$ and immersed into $250 \mathrm{~mL}$ of $\mathrm{HCl}$ buffer $(\mathrm{pH} 1.2)$ for a period of $48 \mathrm{~h}$. The receptor phase was stirred and the temperature was maintained at $37^{\circ} \mathrm{C}$. At predetermined intervals $(0$, $0.5,1,1.5,2,3,4,6,8,10,12,24$ and $48 \mathrm{~h}$ ), samples were drawn from the receptor compartment and equal volumes of fresh media were replaced to maintain sink equilibrium. The amount of drug dissolved was quantitated by high pressure liquid chromatography at $220 \mathrm{~nm}$.

\section{Cytotoxicity studies}

Human multiple myeloma U266 cells were obtained from National center for cellular sciences (NCCS), Pune, India. Minimal essential medium (MEM) was used to culture the cells which was further supplemented with 10\% fetal bovine serum (FBS), 3\% L-glutamine, 
$100 \mathrm{U} / \mathrm{mL}$ pencillin-G and $100 \mu \mathrm{g} / \mathrm{mL}$ streptomycin (Himedia) in a humidified atmosphere of $5 \% \mathrm{CO}_{2}: 95 \%$ air at $37 \pm 2^{\circ} \mathrm{C}$. A 96 well microtiter plate was seeded with 1lakh cells per milliliter of the U266 cell suspension for performing the cytotoxicity assay. After $24 \mathrm{~h}$ of seeding, fresh medium containing different concentrations of LND-PLGA-NPs suspension was added to the plate. A $10 \mathrm{mg} / \mathrm{mL}$ working concentration was freshly obtained by dissolving $0.5 \mathrm{mg}$ of nanoparticles in $4.5 \mathrm{~mL}$ of DMSO and was filtered through a $0.22 \mu$ filter prior to each assay. Blank cells (cells without test samples) were incubated with DMSO whose negligible presence in the wells was not found to be affecting the interfering with experiments.

\section{In vivo bioavailability studies (Ma et al., 2012)}

All the animal investigations were performed as per the requisite protocol approved by the Institutional Animal Ethical Committee of JSS College of Pharmacy, Ooty, India. Approval letter no (JSSCP/IAEC/M.PHARM/ PH.ANALYSIS/02/2012-13). In vivo studies were carried out in healthymale Wistar rats of 150-200g.The animals were housed in individual cages in the animal house for 10 days prior to the initiation of the study to facilitate environmental acclimatization and had access to feed and water ad libitum. $12 \mathrm{~h}$ circadian rhythms were maintained and the temperature was kept constant throughout the study period. The animals were divided into 3 groups containing three animals in each group. The animal dose $(30 \mathrm{mg} / \mathrm{kg}$ ) was chosen based on the surface area ratio from human to rat. Group 1 pertained to the control, group 2 were treated with free drug and group 3 were treated with LNDPLGA-NPs.Blood samples, each not more than $500 \mu \mathrm{L}$ were withdrawn from the tail vein at $0,0.5,1,2,3,4,6,8,10,12,24$ and $48 \mathrm{~h}$ period using a sterilized syringe. Fasting blood samples ( $0^{\text {th }}$ hour) were withdrawn early in the morning. The blood samples were collected in ria-vials containing anticoagulant $(100 \mu \mathrm{L}$ of $11 \%$ sodium citrate) and centrifuged at $4000 \mathrm{rpm}$ for 15 min to separate the plasma and was stored at $-20^{\circ} \mathrm{C}$. The plasma samples were deproteinized by mixing the samples with equal volumes of $10 \%$ perchloric acid and vortexed for $2 \mathrm{~min}$ followed by centrifugation at $4000 \mathrm{rpm}$ for 15 min. The supernatant liquid was separated and analyzed. The amount of LND in plasma samples by was estimated using HPLC at optimized chromatographic conditions.

\section{Statistical analysis}

Statistical data were analyzed using GraphPad Prism $^{\circledR} 6$ program (Graph pad Inc., USA).

\section{In vitro}

The experiments were replicated at least three times. The amount of each drug in the receptor compartment was estimated through HPLC. Data were shown as mean \pm S.D $(n=3)$. The differences between two samples were determined by student's t-test $(\mathrm{p}<0.05)$.

\section{In vivo}

The experiments were replicated at least six times. The amount of drug in plasma was estimated through HPLC. Data was analyzed by one-way analysis of variance (ANOVA), and the mean differences between groups were considered to be significant at $p<0.05$. Data were shown as mean \pm S.D $(n=6)$. The relative oral bioavailability of NFV was calculated according to the equation:

$$
\text { Relative BA }(\%)=100 \times \frac{\left(A U C_{A} / \text { dose }_{B}\right)}{\left(A U C_{B} / \text { dose }_{A}\right)}
$$

$\mathrm{AUC}_{\mathrm{A}}$ and $\mathrm{AUC}_{\mathrm{B}}$ represent the area under the blood concentration time curve of LND-PLGA-NPs and LND suspension, and dose $_{\mathrm{A}}$ and dose $\mathrm{B}_{\mathrm{B}}$ mean the dose of LND-PLGA-NPs and LND suspension following oral administration.

\section{RESULTS AND DISCUSSION}

\section{Preparation}

The nanoparticles containing LND were successfully obtained by the nanoprecipitation method. Different batches of LND-PLGA-NPs were prepared using different concentrations (100-500 mg) of polymer by keeping drug concentration constant with varying stirring rates (500$1500 \mathrm{rpm})$. During the procedure, polymer concentration increment caused a dramatic increase in size of the NPs $(142-265 \mathrm{~nm})$ and entrapment efficiency (16-78\%). But after reaching a concentration of $300 \mathrm{mg}$, the saturation rate and entrapment of the drug to that of polymer were decreased. In the other hand, the stirring rate showed a significant influence on the size of NPs, i.e., at higher stirring rate $(1500 \mathrm{rpm})$ the NPs particle size was reduced, whereas at lower stirring rate the particle size of NPs was not reduced, effectively. Drug loading has a very important influence in the polymeric NPs preference over others such as solid lipid NPs. The improper entrapment leads to the initial burst of the NPs, which hinders its sustained release property (Venkatesh et al., 2015). Hence, the batch with highest entrapment efficiency $(78 \pm 0.92 \%)$ and drug loading $(32 \pm 0.37 \%)$ was chosen as formulation of choice for further characterization. 


\section{Particle size and zeta potential analysis}

The mean particle size of LND NPs was found to be $179.4 \pm 0.9 \mathrm{~nm}$ (Figure $3 \mathrm{a}$ ) and the zeta potential were found to be $-24.4 \pm 0.2 \mathrm{mV}$ (Figure $3 \mathrm{~b}$ ). The experiment was performed in triplicate $(n=3)$ in order to ensure reproducibility and minimize the error. PDI values were found to be 0.464 , which indicates that the system has a relatively narrow distribution. This narrow distribution ensures good stability of PLGA-NPs suspension by avoiding problems such as Ostwald ripening. Zeta potential reports had showed that the prepared PLGA nanoparticles had typical negative zeta potential attributed to PLGA preparations due to carboxyl groups present in their structure. The Zeta potential of the prepared NPs is also a prominent factor to ensure stability. Highly charged NPs are capable to remain stable as colloidal suspension. Since the prepared NPs have shown high zeta potential which ensure the stability of the formulation. The morphological evaluation (size, shape and morphology) of the LND-PLGA-NPs was performed using SEM (Figure 4). SEM studies confirm that nanoparticles are in the range of 50-300 nm with smooth surface and spherical shape which ensures the drug release in sustained manner.

$\begin{array}{rlllll}\text { (A) } & & & \text { Size (d.nm): } & \text { \% Intensity: } & \text { St Dev (d.n... } \\ \text { Z-Average (d.nm): } & 179.4 & \text { Peak 1: } & 292.2 & 96.0 & 216.5 \\ \text { Pdl: } & 0.464 & \text { Peak 2: } & 4660 & 4.0 & 806.5 \\ \text { Intercept: } & 0.943 & \text { Peak 3: } & 0.000 & 0.0 & 0.000 \\ \text { Result quality : } & \text { Good } & & & & \end{array}$

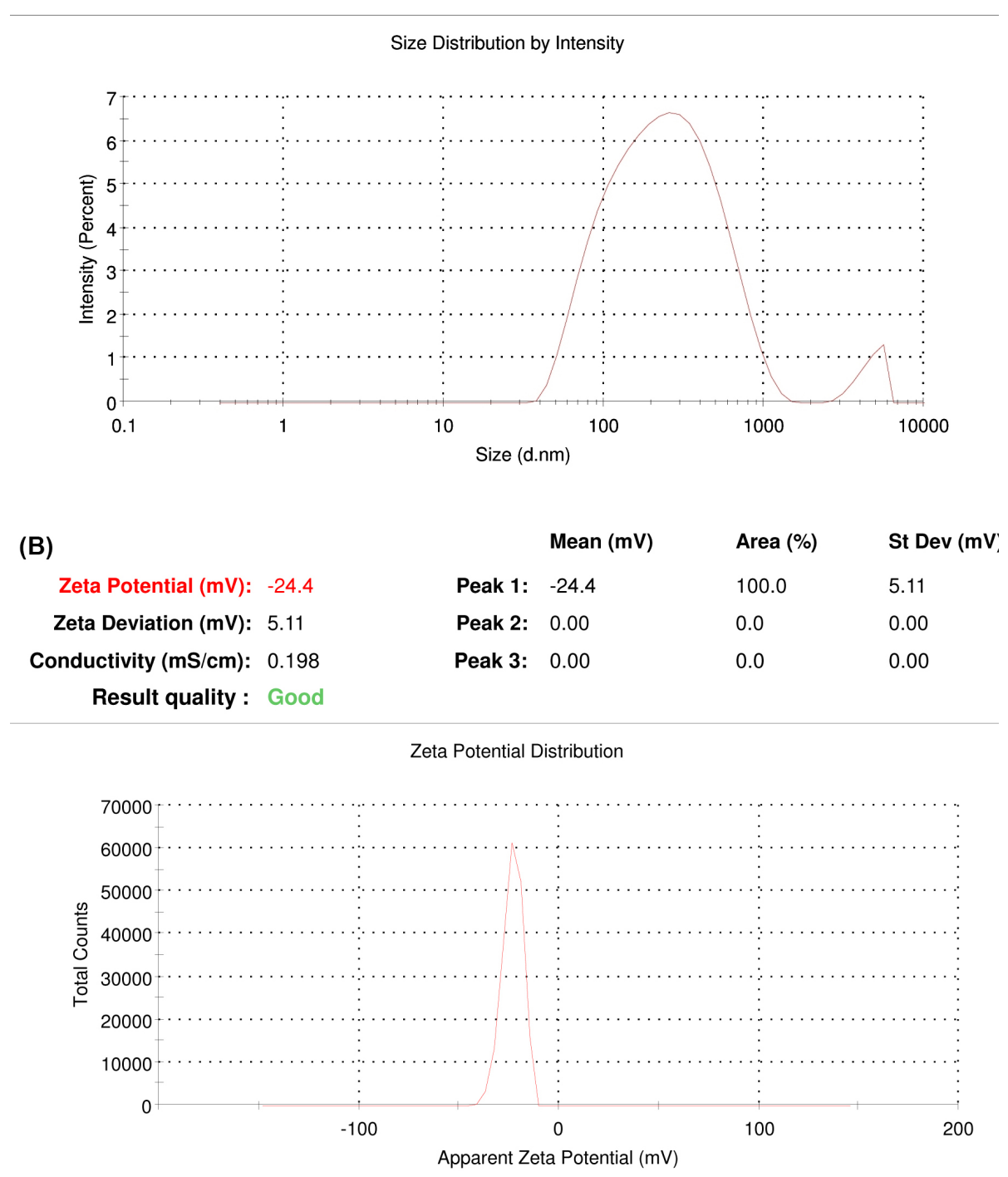

FIGURE 3 - (a) Particle Size; (b) Zeta potential of lenalidomide-PLGA-NPs. 


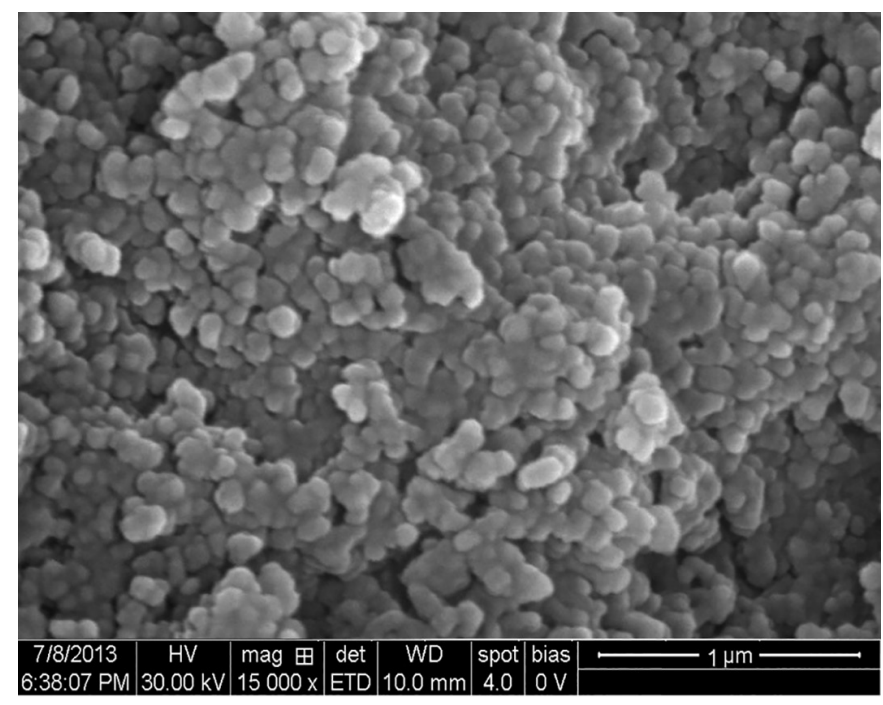

FIGURE 4 - SEM photograph of lenalidomide -PLGA-NPs.

\section{DSC and XRD studies}

The DSC thermograms of LND, PLGA, and NPs are shown in Figure 5. The DSC curve of LND exhibit an exothermic peak at peak temperature of $265^{\circ} \mathrm{C}$ corresponding to its melting point. The polymer, PLGA had shown peak at a temperature of 65.12. As per the DSC graph of LND-PLGA-NPs, the characteristic exothermic peak of LND was observed with minimal intensity and shifted to lower temperature range. This indicates the conversion of crystalline nature of LND to amorphous form which ensures the better stability. Furthermore, it also confirms that LND was entrapped into PLGA-NPs. Reported findings have suggested that with an increase in the amorphous nature of the therapeutic system, a corresponding increase in the efficiency of delivery is observed (Abdelwahed et al., 2006).

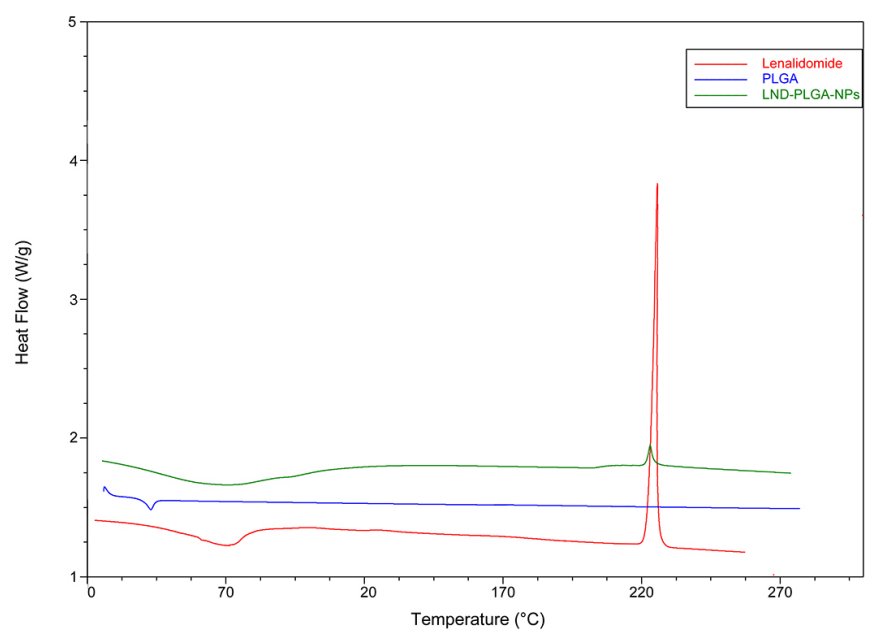

FIGURE 5 -DSC curves of lenalidomide, PLGA, and lenalidomide -PLGA-NPs.
The physical nature of pure LND, PLGA-NPs and LND loaded PLGA-NPs were observed using X-ray diffraction analysis (Figure 6). Pure LND possessed a crystal structure when compared to LND-PLGA-NPs, which were in amorphous form. Pure LND has shown multiple peaks at different $2 \theta$ values indicating crystalline form of pure LND. However, the intensity of LND peaks were greatly reduced in PLGA-NPs signifying the conversion of crystalline form LND to amorphous form and LND loading in to PLGA-NPs.

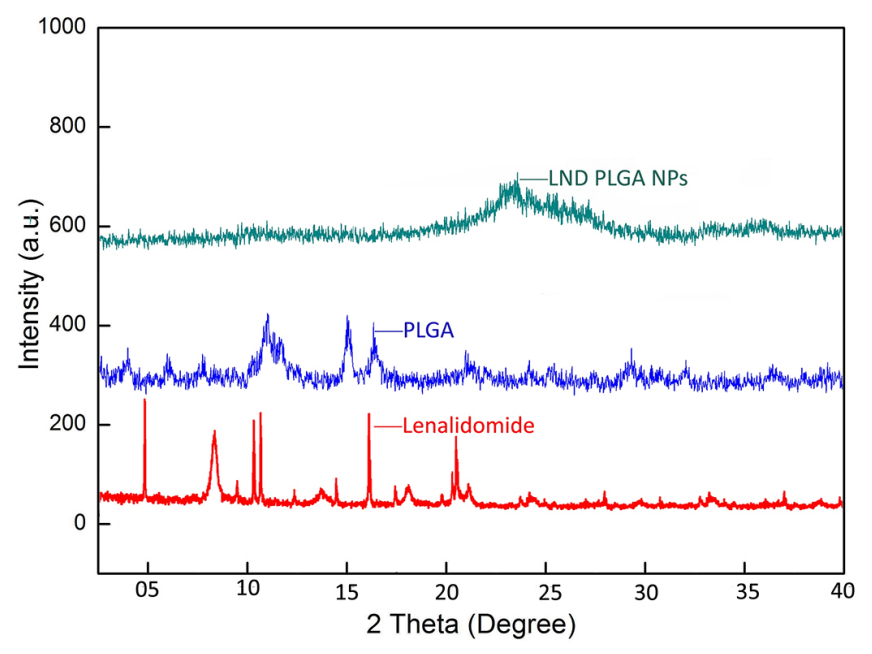

FIGURE 6 -XRD of lenalidomide, PLGA and lenalidomidePLGA-NPs.

\section{In vitro drug release}

In vitro release studies of LND loaded PLGA-NPs were studied up to $48 \mathrm{~h}$ in $\mathrm{HCl}$ buffer ( $\mathrm{pH}$ 1.2) for a period of $48 \mathrm{~h}$. The summarized in vitro release of LND-PLGANPs is shown in Figure 7, as cumulative percentage drug release. The NPs has shown a biphasic pattern of drug release. Initially, a burst release of drug was observed (39\%) followed by sustained release. $40 \%$ of LND was released within 6 hours and about $76.89 \pm 2.99 \%$ and 83 $\pm 3.90 \%$ was released in 24 and $48 \mathrm{~h}$ respectively. This clearly depicts the pronounced time prolongation of the drug release. The initial burst release of drug may be due to drug desorption of surface entrapped or adhered LND from the particle surface, and the sustained release can be characterized by the drug diffusion through the polymeric matrix and subsequent dissolution of drug or erosion of the polymeric matrix. In dissolution media, the release of LND from PLGA-NPs occurred either by the formation of holes in the particles or by their disintegration. A constant rate of release of the drug from the NPs will facilitate better drug control in vivo. 


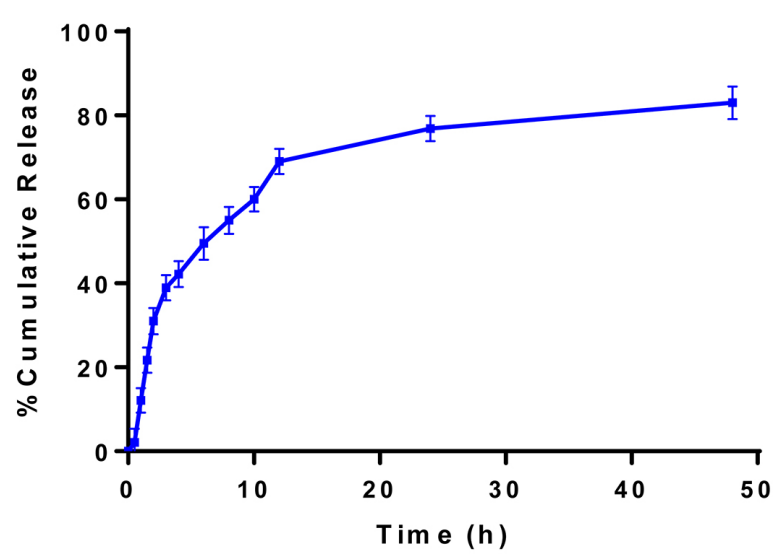

FIGURE 7 -Release rates of lenalidomide from PLGA-NPs in vitro in $\mathrm{HCl}$ buffer $\mathrm{pH} 1.2($ mean $\pm \mathrm{SD}, \mathrm{n}=3)$.

\section{Cytotoxicity studies}

The extent of cell viability due to LND-PLGANPs was examined by MTT assay in U266 cells. Eight concentrations (1-100 $\mu \mathrm{g} / \mathrm{mL}$ ) of LND-PLGA-NPs were prepared and tested yielding proof that the NPs exhibited significant toxicity against U266 cells. Cytotoxicity concentration $\left(\mathrm{CTC}_{50}\right)$ value for NPs was calculated from the concentration and subsequent response; it was found to be $34.09 \mu \mathrm{g} / \mathrm{mL}$ (Figure 8 ). This proves the cytotoxic potential of the PLGA-NPs and lends further credence to the viability if this concept.

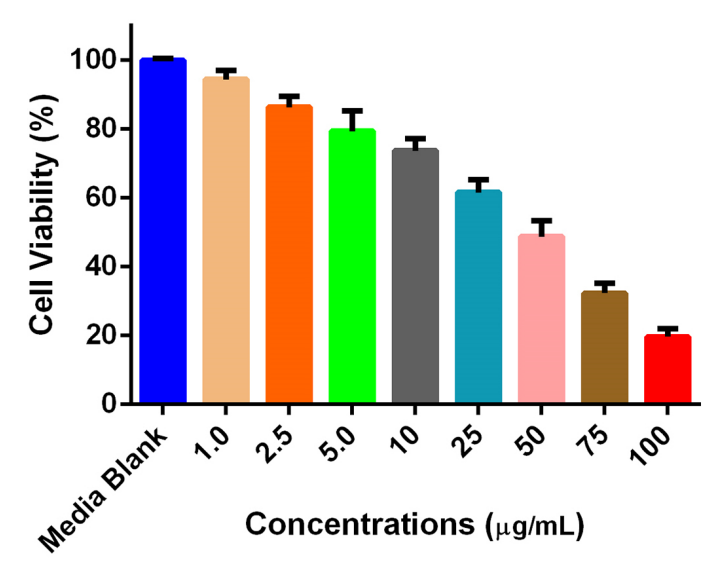

FIGURE 8 - MTT assay of lenalidomide - PLGA-NPs.

\section{In vivo bioavailability studies}

The formulations (LND aqueous suspension, dispersion of the LND-loaded PLGA NPs) were administered by oral gavage with a single dose of 30 $\mathrm{mg} / \mathrm{kg}$. The nanoparticles were dispersed in ultra-pure water. Table I and Figure 9 shows the pharmacokinetic data of the drug suspension and nanoparticles. Figure 9 depicts a remarkable difference in bioavailability between LND PLGA NPs and LND suspension. After oral administration of LND suspension, the drug was rapidly absorbed and a $\mathrm{C}_{\max }$ of $0.57 \pm 0.003 \mu \mathrm{g} /$ $\mathrm{mL}$ was reached in $1.5 \mathrm{~h}$. Consequently, the plasma concentration decreased abruptly, as the drug was rapidly distributed and metabolized and was detected up to $48 \mathrm{~h}$ after administration, resulting in low $\mathrm{AUC}_{0-\mathrm{t}}$ $(4.9695 \pm 0.036 \mathrm{~h} . \mu \mathrm{g} / \mathrm{mL})$ and low $\mathrm{t}_{1 / 2}(9.762 \pm 1.9 \mathrm{~h})$. Figure 9 shows that peak plasma concentration for LND PLGA NPs $\left(\mathrm{C}_{\max }\right)$ of approximately $1.37 \pm 0.007 \mu \mathrm{g} / \mathrm{mL}$ was achieved at $4 \mathrm{~h}$.

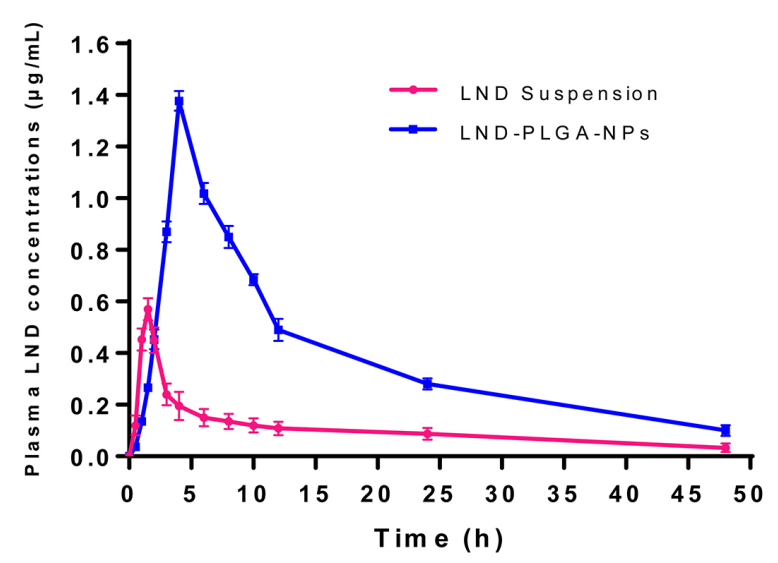

FIGURE 9 - Mean plasma concentration time profiles of lenalidomide in wistar rats: (a) lenalidomide suspension (b) lenalidomide PLGA-NPs with (mean $\pm \mathrm{SD}, \mathrm{n}=6$ ).

Table I depicts the pharmacokinetic parameters $\mathrm{C}_{\max }, \mathrm{AUC}_{0-\mathrm{t}}, \mathrm{t}_{1 / 2}$ and $\mathrm{t}_{\max }$ after oral administration of LND loaded PLGA-NPs and suspension. From Table I, it can be perceived that, the $\mathrm{C}_{\max }$ value for LND PLGA NPs (1.377 \pm 0.007$)$ was significantly higher than LND suspension $(0.57 \pm 0.003)$. In the same way, the $t_{1 / 2}$ was rapid for LND-PLGA-NPs (20.2183 \pm 2.2$)$, when compared to LND suspension $(9.762 \pm 1.9)$. From the data in Table I, it is apparent that, the $t_{\max }$ was also rapid for LND PLGA NPs (4.0 \pm 0.026$)$, when compared to LND suspension $(1.5 \pm 0.024)$. This may be due to the primarily related prolonged absorption phase and sustained release of PLGA-NPs. Likewise, the area under curve AUC for LND PLGA NPs was 3-fold higher than the LND suspension. Finally, the relative bioavailability for LND PLGA NPs was computed and found to be $367.80 \%$, suggesting better absorption of LND-PLGA-NPs compared to the suspension. On the contrary, particles belonging to nanoscale are easily absorbed into the intestinal folds, while larger particle surface area to volume offers faster 
TABLE I - Pharmacokinetic parameters of lenalidomide after oral administration of LND loaded PLGA-NPs and suspension at the dose of $30 \mathrm{mg} / \mathrm{kg}$

\begin{tabular}{ccc}
\hline Parameters & LND Suspension & LND-PLGA-NPs \\
\hline $\mathbf{C}_{\max }(\mu \mathrm{g} / \mathrm{mL})$ & $0.57 \pm 0.003$ & $1.377 \pm 0.007$ \\
$\mathbf{A U C}_{\mathbf{0 - \mathrm { t }}}(\mathrm{h} . \mu \mathrm{g} / \mathrm{mL})$ & $4.9695 \pm 0.036$ & $18.276 \pm 0.042$ \\
$\mathbf{t}_{1 / 2}(\mathrm{~h})$ & $9.762 \pm 1.9$ & $20.2183 \pm 2.2$ \\
$\mathbf{t}_{\max }(\mathrm{h})$ & $1.5 \pm 0.024$ & $4.0 \pm 0.026$ \\
$\mathbf{F}_{\text {rel }}(\%)$ & - & 367.80 \\
\hline
\end{tabular}

drug dissolution. In current study, there was a significant improvement in bioavailability with LND-PLGA-NPs in contrast to LND suspension. The reasons may be many, but it is assumed that nano based drug delivery system enhances the bioavailability by modulating the physiochemical properties of poorly soluble drugs. Overall, our results suggest that LND-PLGA-NPs are most likely to have great potential as a therapeutic, by enhanced pharmacokinetic profiles.

\section{CONCLUSION}

Nanoparticles of a poorly soluble drug LND were successfully formulated by nano-precipitation method using PLGA as polymer. The particle size, zeta and SEM reports confirmed that the size of LNDPLGA-NPs was below $200 \mathrm{~nm}$ with spherical shape and uniform size distribution. DSC and XRD analysis of NPs confirmed the conversion of the crystalline nature of LND to amorphous form and drug entrapment within the NPs. Cytotoxic studies suggested that LND-PLGANPs were toxic towards U266 cancer cells. In vitro and in vivo studies confirmed that LND-PLGA-NPs demonstrate increased bioavailability with a sustained release up to $48 \mathrm{~h}$. Due to their hydrophobic nature, conventional chemotherapeutics of LND suffer from poor solubility and an inability to penetrate the tumors (poor bioavailability). This results in grave side effects that include immune system depletion and metastasis to neighboring organs. Hence, the present PLGA nanoparticles can effectively solve the solubility problem and enhance the bioavailability of LND.

\section{ACKNOWLEDGEMENT}

The authors sincerely thank Natco Pharma Ltd, Hyderabad, India for providing lenalidomide and thalidomide as gift samples.

\section{REFERENCES}

ABDELWAHED, W.; DEGOBERT, G.; STAINMESSE, S.; FESSI, H. Freeze-drying of nanoparticles: formulation, process and storage considerations. Adv. Drug Deliv. Rev., v. 58, n. 15, p.1688-1713, 2006.

EGUSQUIAGUIRRE, S.; IGARTUA, M.; HERNÁNDEZ, R.; PEDRAZ, J., Nanoparticle delivery systems for cancer therapy: advances in clinical and preclinical research. Clin. Transl. Oncol., v.14, n.2, p.83-93, 2012.

FOOD AND DRUG ADMINISTRATION. FDA. Guidance for industry: bioanalytical method validation. US Department of Health and Human Services. Silver Spring, MD: Food and Drug Administration, Center for Drug Evaluation and Research (CDER), Center for Veterinary Medicine (CV), 2001. p.1-22.

GOMATHI, T.; GOVINDARAJAN, C.; ROSE, H.R, M.H.; SUDHA, P.N.; IMRAN, P.K.M.; VENKATESAN, J.; KIM, S.-K. Studies on drug-polymer interaction, in vitro release and cytotoxicity from chitosan particles excipient. Int. $J$. Pharm., v.468, n.2, p.214-222, 2014.

KARRI, V.V.S.N.; RAMAN, S.; KUPPUSAMY, G.; MULUKUTLA, S.; RAMASWAMY, S.; MALAYANDI, R. Terbinafine hydrochloride loaded nanoemulsion based gel for topical application. J. Pharm. Invest., v.45, n.1, p.79-89, 2015.

KASTRITIS, E.; DIMOPOULOS, M.A. The evolving role of lenalidomide in the treatment of hematologic malignancies. Expert Opin Pharmacother., v.8, n.4, p.497-509, 2007.

MA, Y.; ZHAO, X.; LI, J.; SHEN, Q. The comparison of different daidzein-PLGA nanoparticles in increasing its oral bioavailability. Int. J. Nanomed., v.7, n.1, p.559-570, 2012. 
MAEDA, H.; WU, J.; SAWA, T.; MATSUMURA, Y.; HORI, $\mathrm{K}$. Tumor vascular permeability and the EPR effect in macromolecular therapeutics: a review. J. Control. Rel., v.65, n.2, p.271-284, 2000.

MANNEMALA, S.S.; NAGARAJAN, J.S.K. Development and validation of a generic liquid chromatographic method for the simultaneous determination of five commonly used antimalarial drugs: application to pharmaceutical formulations and human plasma. J. Sep. Sci., v.38, n.9, p.1521-1528, 2015a.

MANNEMALA, S.S.; NAGARAJAN, J.S.K. Development and validation of a HPLC-PDA bioanalytical method for the simultaneous estimation of Aliskiren and Amlodipine in human plasma. Biomed. Chromatogr., v.29, n.3, p.346$352,2015 b$.

MORGENROTH, A.; DINGER, C.; ZLATOPOLSKIY, B.D.; AL-MOMANI, E.; GLATTING, G.; MOTTAGHY, F.M.; RESKE, S.N. Auger electron emitter against multiple myeloma targeted endo-radio-therapy with 125I-labeled thymidine analogue 5-iodo-4'-thio-2'-deoxyuridine. Nucl. Med. Biol., v.38, n.7, p.1067-1077, 2011.

NOORI KOOPAEI, M.; KHOSHAYAND, M.R.; MOSTAFAVI, S.H.; AMINI, M.; KHORRAMIZADEH, M.R.; JEDDI TEHRANI, M.; ATYABI, F.; DINARVAND, R. Docetaxel loaded PEG-PLGA nanoparticles: optimized drug loading, in-vitro cytotoxicity and in-vivo antitumor effect. Iranian J. Pharm. Res., v.13, n.3, p.819-833, 2014.
RICHARDSON, P.G.; MITSIADES, C.; HIDESHIMA, T.; ANDERSON, K.C. Lenalidomide in multiple myeloma. Expert Rev. Anticancer Ther., v.6, n.8, p.1165-1173, 2006.

SONG, J.-X.; YAN, Y.; YAO, J.; CHEN, J.-M.; LU, T.-B. Improving the solubility of lenalidomide via cocrystals. Cryst. Growth Des., v.14, n.6, p.3069-3077, 2014.

VEGA, E.; GAMISANS, F.; GARCÍA, M.L.; CHAUVET, A.; LACOULONCHE, F.; EGEA, M.A. PLGA nanospheres for the ocular delivery of flurbiprofen: drug release and interactions. J. Pharm. Sci., v.97, n.12, p.5306-5317, 2008.

VENKATESH, D.N.; BASKARAN, M.; KARRI, V.V.S.R.; MANNEMALA, S.S.; RADHAKRISHNA, K.; GOTI, $\mathrm{S}$. Fabrication and in vivo evaluation of Nelfinavir loaded PLGA nanoparticles for enhancing oral bioavailability and therapeutic effect. Saudi Pharm. J., v.23, n.6, p.667-674, 2015.

Received for publication on $29^{\text {th }}$ September 2015 Accepted for publication on $06^{\text {th }}$ October 2016 